\title{
Hemodynamic Instability during Acute Kidney Injury and Acute Renal Replacement Therapy: Pathophysiology and Clinical Implications
}

\author{
Gianluca Villa $^{a}$ Faeq Husain-Syed ${ }^{b}$ Thomas Saitta ${ }^{a}$ Dario Degl'Innocenti ${ }^{a}$ \\ Francesco Barbanic Marco Restad $^{\mathrm{d}}$ Gianluca Castellani ${ }^{\mathrm{e}}$ Stefano Romagnoli ${ }^{\mathrm{a}}$ \\ aDepartment of Health Science, Section of Anesthesiology and Intensive Care, University of Florence, Florence, \\ Italy; ${ }^{b}$ Division of Nephrology, Department of Internal Medicine II, Pulmonology and Critical Care Medicine, \\ University Hospital Giessen and Marburg, Giessen, Germany; 'Department of Anesthesia and Intensive Care, \\ Section of Oncological Anesthesia and Intensive Care, Azienda Ospedaliero Universitaria Careggi, Florence, Italy; \\ dU.O. Anestesia e Rianimazione Polispecialistica - IRCCS Policlinico San Donato, Milan, Italy; ${ }^{\text {eDepartment of }}$ \\ Anesthesia and Intensive Care, Humanitas Clinical and Research Center - IRCCS, Milan, Italy
}

\section{Keywords}

Blood pressure - Cardiac output · Hypotension .

Inflammation · Systemic vascular resistance

\begin{abstract}
Hemodynamic instability associated with acute renal replacement therapy (aRRT, HIRRT) and/or with acute kidney injury (AKI) is frequently observed in the intensive care unit; it affects patients' renal recovery, and negatively impacts short- and long-term mortality. A thorough understanding of mechanisms underlying HIRRT and AKI-related hemodynamic instability may allow the physician in adopting adequate strategies to prevent their occurrence and reduce their negative consequences. The aim of this review is to summarize the main alterations occurring in patients with $\mathrm{AKI}$ and/or requiring aRRT of those homeostatic mechanisms which regulate hemodynamics and oxygen delivery.

In particular, a pathophysiological approach has been used to describe the maladaptive interactions between cardiac output and systemic vascular resistance occurring in these
\end{abstract}

karger@karger.com

(c) 2021 S. Karger AG, Basel

www.karger.com/bpu

Karger" patients and leading to hemodynamic instability. Finally, the potential positive effects of aRRT on these pathophysiological mechanisms and on restoring hemodynamic stability have been described.

(c) 2021 S. Karger AG, Basel

\section{Introduction}

Hemodynamic instability is frequently observed during critical illness. It has a major impact on patients' outcomes in the intensive care unit (ICU), mainly interfering with tissue perfusion and oxygen delivery, thus leading to multiorgan dysfunction [1]. Similarly, acute kidney injury (AKI) frequently develops in critically ill patients, further affecting patients' outcomes [2]. Hypotension and AKI are associated through direct (causative) and indirect mechanisms (see Fig. 1, 2). In particular, beyond hypoperfusion-induced kidney injury and shock, AKI and hypotension can be contemporaneous clinical expressions of a single underlying cause of organ damage 


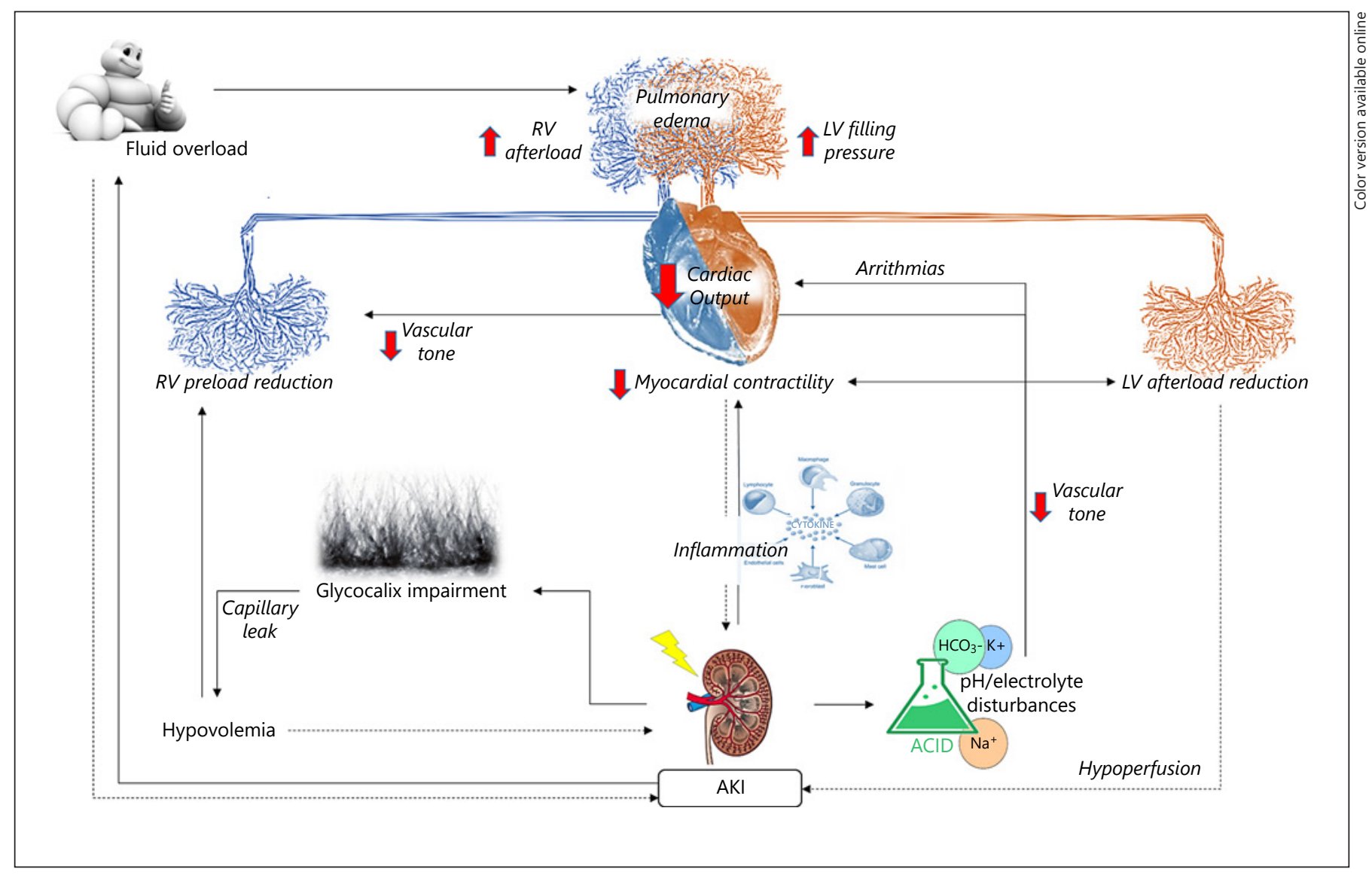

Fig. 1. Hemodynamic instability during critical illness and development of AKI. CO, cardiac output; AKI, acute kidney injury; LV, left ventricular; RV, right ventricular.

[3]. Interestingly, $10-20 \%$ of patients with AKI in the ICU require acute renal replacement therapy $(\mathrm{aRRT})$, and their expected mortality rate is almost 50\% [4]. A clear epidemiological and pathophysiological connection is also recognized between hypotension and aRRT. An exact definition of hemodynamic instability during renal replacement therapy (HIRRT) is lacking. Although the association between HIRRT and adverse short- and longterm outcomes is unclear and mainly extrapolated from the ESRD population with maintenance hemodialysis, reports on critically ill patients associate HIRRT with a higher mortality rate and impaired renal recovery [5].

Considering the impact of a pathophysiological approach to treatment in the ICU, this review describes the pathophysiological mechanisms of hemodynamic instability among patients with AKI and in patients requiring aRRT. Furthermore, we analyze potential positive effects of RRT on these pathophysiological mechanisms and on restoring hemodynamic stability.

\section{Pathophysiology of Hemodynamic Instability during AKI and RRT}

Hemodynamics and oxygen delivery are tightly regulated by homeostatic mechanisms, which are mainly dependent on the adaptive interaction between cardiac output (CO) and systemic vascular resistance (SVR) due to a well-functioning endothelial barrier. Reduced CO and/or impaired SVR are fundamental pathophysiological mechanisms that underpin hemodynamic instability, particularly during endothelial dysfunction [6].

\section{Reduction in $\mathrm{CO}$}

$\mathrm{CO}$ is influenced by heart rate, ventricular preload, contractility, and ventricular afterload. Generally, a reduction in heart rate, ventricular preload, and contractility leads to a decrease in $\mathrm{CO}$, which is similar to the effect of an increase in ventricular afterload. Alterations in these parameters lead to kidney hypoperfusion, impairment, 


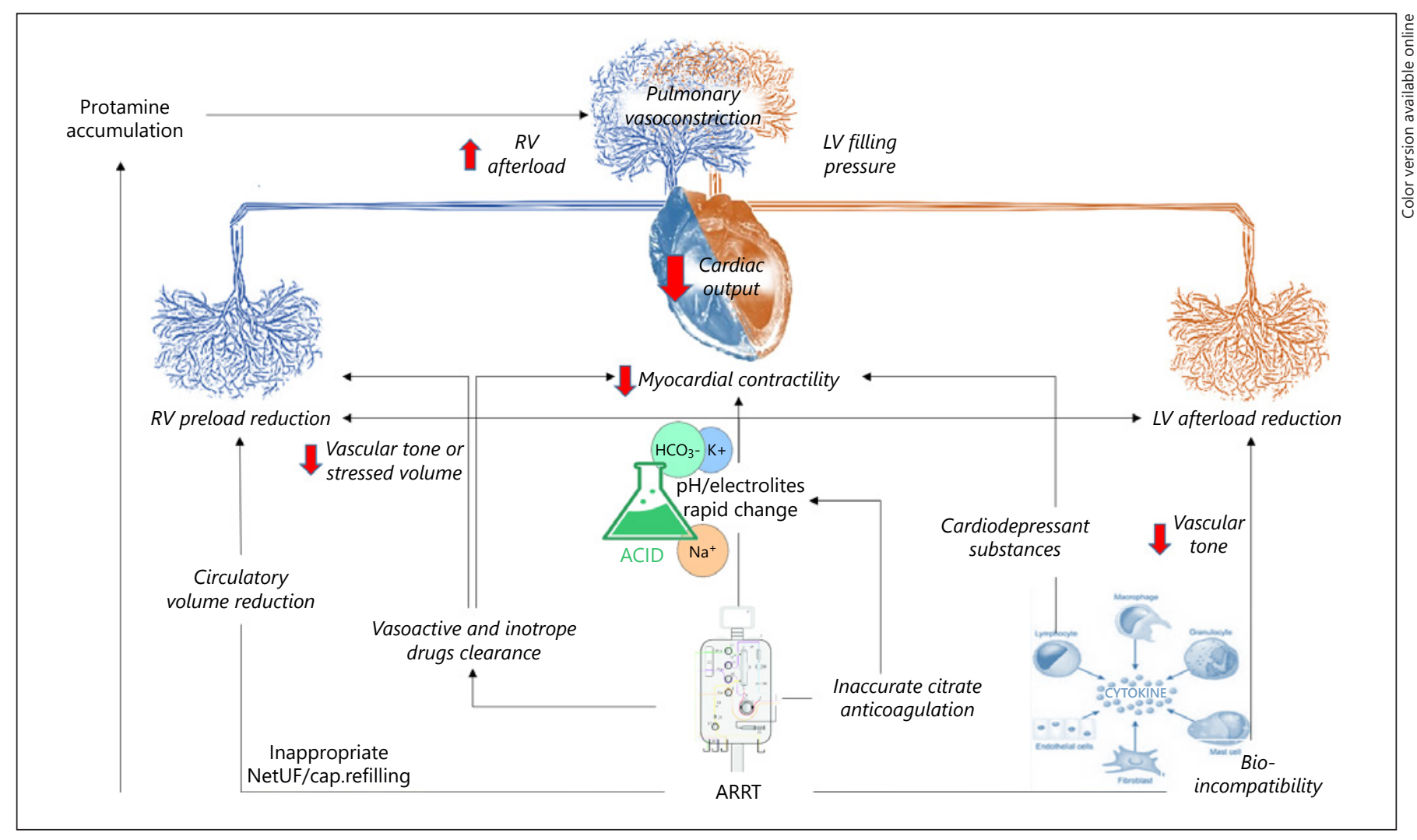

Fig. 2. aRRT and hemodynamic instability in critical illness. aRRT, acute renal replacement therapy; LV, left ventricular; RV, right ventricular; NetUF, net ultrafiltration.

and dysfunction. Equally, these parameters can be impaired during AKI and aRRT, leading to hemodynamic instability.

Ventricular preload refers to the tension observed in the ventricle just before contraction and mainly depends on ventricular end-diastolic volume. Clinically, ventricular preload represents venous return and thus depends on atrial pressure, mean systemic filling pressure, and resistance to venous return. Impaired ventricular preload may lead to AKI via several mechanisms. Equally, AKI and/or aRRT may affect ventricular preload, leading to hemodynamic instability. Two main scenarios can manifest in this context:

\section{Reduction in Preload due to Absolute or Relative \\ Hypovolemia}

An acute impairment in preload can occur during an absolute reduction in effective circulating volume (e.g., during hypovolemia and/or massive bleeding leading to hypovolemic shock) and/or during a maladaptive increase in venous capacitance (e.g., during sepsis or hepa- torenal syndrome leading to distributive shock). In these circumstances, kidney hypoperfusion is recognized as the main pathophysiological mechanism of AKI. This is particularly true in patients with an impaired intrarenal vascular autoregulation capacity, even in the absence of renal blood flow reduction, such as in patients with sepsis [7]. Interestingly, a reduction in preload and AKI can contemporaneously occur from a single common causative factor (see Fig. 1). Systemic inflammation, for example, is as an important pathophysiological mechanism leading to venodilation (i.e., a reduction in preload occurs due to relative hypovolemia) and kidney impairment via inflammatory mechanisms [7]. Most of these complex clinical scenarios are observed with cardiorenal syndrome type 5 [8].

In addition, aRRT might be associated with hemodynamic instability due a reduction in venous return and $\mathrm{CO}$ (see Fig. 2). In particular, prolonged or excessive net ultrafiltration (NetUF) can result in intravascular volume depletion if not adequately compensated by capillary refilling. This is particularly true for patients undergoing 
intermittent hemodialysis (IHD) and in patients with microcirculatory impairment. This is also applicable in patients who, despite hypervolemia, have a reduced effective blood volume (e.g., patients with chronic heart failure) or impaired venous return (e.g., patients on mechanical ventilation) [9].

\section{Increased Preload and Cardiac Dysfunction}

\section{Associated with Volume Overload}

Hypervolemia is frequently observed during critical illness, and ceteris paribus severely affects patients' outcomes [10]. Volume overload and tissue edema, particularly in the abdomen, might reduce kidney perfusion pressure, leading to kidney impairment and dysfunction [11]. Heart failure and increased end-diastolic volume often lead to kidney hypoperfusion. Indeed, acute right ventricular dysfunction, which can cause hemodynamic instability, might reduce venous return, leading to congestive heart failure and kidney dysfunction. The increase in central venous pressure and consequent increase in pressure in the venous splanchnic system, renal venous system, and efferent arterioles severely impairs glomerular filtration pressure and urine production. Under these conditions, kidney dysfunction occurs, even in absence of kidney hypoperfusion. Conversely, kidney dysfunction leading to volume overload and hypervolemia may contribute to a worsening in cardiac function, further enhancing the epidemiological link between AKI and hemodynamic instability. Cardiorenal syndrome type 3 is a clear example of hemodynamic instability caused by kidney dysfunction, hypervolemia, and congestive heart failure (see Fig. 1) [12].

Myocardial rhythm and contractility can be dysregulated during both AKI and aRRT. Acid-base and electrolyte alterations, solute and neuroendocrine dysregulation, and pathologic pressure within myocardial chambers are all pathophysiologic mechanisms that lead to inotropic and chronotropic dysfunction (see Fig. 1, 2) $[5,11])$. Similarly, hemodynamic instability due to heart failure may lead to AKI via several mechanisms [8].

AKI often induces metabolic acidosis, which can directly impair cardiac function, leading to a proarrhythmic state and a reduction in contractility. Acidosis affects the myocyte action potential, which in turn influences the transmembrane flux of electrolytes and contributes to a proarrhythmic state. Moreover, acidosis reduces the calcium sensitivity of the contractile proteins and leads to impaired $\beta$-adrenergic receptor expression, further reducing myocardial contractility [13].
Sporadically, acid-base disorders may also be observed during aRRT, especially in patients undergoing regional citrate anticoagulation (RCA) [14]. Acid-base disorders mainly occur due to (1) an inadequate amount of buffer during treatment or (2) an impaired citrate metabolism. The citrate load infused during CRRT is promptly metabolized by the liver and myocytes and is fed into the Krebs cycle to produce $3 \mathrm{mmol}$ of bicarbonate for each mmol of trisodium citrate produced. In these circumstances, dialysate containing a low concentration of bicarbonate is usually used. If a low citrate load is infused (e.g., for a reduced citrate dose, reduced blood flow, or a high effluent dose), a negative bicarbonate mass balance might occur, leading to metabolic acidosis. Metabolic acidosis can also occur even if an appropriate citrate load is achieved, particularly if the calcium-citrate complex is not adequately metabolized. In particular, lack of citrate metabolism does not produce bicarbonate and thus leads to a negative buffer balance [14].

Electrolyte disorders are frequently observed during AKI. Hyperkalemia is a potentially life-threatening complication of AKI and is an indication for RRT. Hyperkalemia is associated with potentially fatal arrhythmias and cardiac toxicity, which are caused by a reduction in the resting membrane potential of cells. An increase in the extracellular concentration of potassium limits myocyte repolarization and increases cell excitability, leading to severe arrhythmia [12].

Hypercalcemia usually occurs in severely impaired systemic conditions where AKI also develops (e.g., tumor lysis syndrome and crush syndrome). It can severely affect myocardial electrical conduction, mainly reducing the QT interval [15]. Electrolyte alterations might occur also during aRRT. Hypokalemia might develop during high-dose CRRT and at centers that can only store solutions containing low levels of potassium; thus, potassium can reach harmful concentrations [9].

Hypocalcemia is common in critically ill patients. An ionized calcium concentration of $<1.0 \mathrm{mmol} / \mathrm{L}$ is an independent predictor of all-cause mortality in critically ill patients with AKI requiring aRRT [16]. Moreover, a low dialysate calcium concentration is associated with an increased risk of sudden cardiac arrest in patients undergoing maintenance IHD [17]. Patient treated with RCA are at particular risk of hypocalcemia. The calcium is removed as the citrate-calcium complex is replaced to maintain homeostasis. In these circumstances, inadequate supplementation might cause severe hypocalcemia. Nonetheless, the most harmful condition leading to hypocalcemia during RCA is impaired citrate metabolism. 
If citrate is not metabolized in the Krebs cycle, calcium ions from the calcium-citrate complex cannot be freed; thus, the ionized calcium concentration (i.e., the bioactive form) decreases [14]. Notably, the chelating effect of citrate affects other transmembrane bivalent cations, such as magnesium. Hypomagnesemia is a potentially severe electrolyte disorder that can occur during CRRT, particularly during RCA [18].

Volume overload and hypervolemia may lead to cardiac arrhythmia, mainly because a sudden increase in ventricular volume causes diastolic stretch, leading to heterogeneous activation of transmembrane ion channels, decreased action potential duration, and alteration of ventricular refractoriness (i.e., the mechanoelectrical feedback). These effects are intensified during sympathetic activation, leading to ventricular ectopy, ventricular tachycardia, or ventricular fibrillation, particularly in patients with repolarization inhomogeneities and intramural scars [19].

Neuroendocrine alterations during AKI may also lead to arrhythmia and heart failure. Cardiotoxic effects due to sympathetic nervous system activation depend on renin-angiotensin-aldosterone system activation, which increases myocardial oxygen demand and affects calcium homeostasis, myocyte hypertrophy, and apoptosis. Renin-angiotensin-aldosterone system activation causes systemic vasoconstriction, which increases afterload; extracellular volume expansion via increased sodium retention, which causes volume overload; and impaired ventricular function, particularly in patients with already impaired ventricular function [12].

Hypothermia frequently develops in patients in the ICU, mainly in those treated with aRRT, and directly correlates with ICU mortality. Continuous veno-venous modalities, dialysate temperature, and dialysate and blood flow are all variables that contribute to the development of hypothermia. Although mild hypothermia is a potential mechanism to prevent hypotension during RRT via systemic vasoconstriction [9], it has also been associated with myocardial stunning and cardiogenic shock. Severe hypothermia $\left(<30^{\circ} \mathrm{C}\right)$ increases the cytoplasmatic concentration of calcium and cyclic adenosine monophosphate, reducing the calcium sensitivity of contractile proteins and causing cell dysfunction and damage [20]. Furthermore, hypothermia impairs the sensitivity of adrenergic receptors to agonists and also affects their pharmacokinetic/pharmacodynamic properties [21].

Several cardiodepressant substances are produced during AKI and RRT. For example, cardiodepressant effects have been demonstrated for uremic toxins, not only during CKD but also during AKI [12]. Pro-inflammatory mediators can also cause acute myocardial dysfunction during AKI. Particularly, cytokines, such as tumor necrosis factor- $\alpha$, interleukin (IL)- $1 \beta$, IL-6, and IL- 18 , are produced during AKI and multiorgan dysfunction, leading to myocardial stunning and cardiogenic shock. This effect might derive from an impairment in cytokine-related myocyte contraction, mitochondrial dysfunction, apoptosis, and fibrosis induction [12]. Interestingly, RRT can induce myocardial stunning and regional ventricular wall motion abnormalities. Several reports describe myocardial stunning during IHD, in both chronically and acutely ill patients [9]. This phenomenon seems to be related to reduced regional myocardial perfusion, even in the absence of atherosclerotic coronary artery disease, or independently from excessive NetUF and hypovolemia.

CRRT, particularly at treatment initiation, is associated with a transient and acute deterioration in regional left ventricular contractility [22]. Although the exact mechanism by which CRRT triggers myocardial stunning still remains incompletely understood, it seems to be linked to microcirculatory alterations and inflammatory mediators generated during bioincompatible reactions. Activated complement components can contribute to myocardial injury under these circumstances via the indirect effects of anaphylatoxins and the direct effects of C5b-9, which modify leukocyte responses, alter vascular homeostasis, lead to cellular activation, and ultimately induce myocardial damage $[23,24]$.

Afterload describes the pressure in the artery just before ventricular ejection; thus, afterload represents the force that the ventricle must overcome to eject blood during systole. In terms of hypotension and hemodynamic instability, an inappropriate increase in right ventricular afterload and a decrease in left ventricular afterload should be considered (see Fig. 1, 2). A maladaptive reduction in left ventricular afterload occurs when inappropriate vascular resistance contributes to hemodynamic instability, such as during distributive shock.

\section{Inappropriate Increase in Right Ventricular Afterload}

Pulmonary hypertension during hypoxemia, pulmonary edema, or mechanical ventilation can increase right ventricular afterload. Consequent right ventricular dysfunction can affect left ventricular preload, leading to hemodynamic instability. Volume overload and pulmonary edema are frequently observed in critically ill patients with AKI, particularly in patients with chronic left ventricular dysfunction. One cause of an increase in right ventricular afterload during RRT is protamine intoxica- 
tion (see Fig. 2). Regional heparin-protamine protocols are uncommon nowadays; however, when applied, particularly in patients undergoing cardiac surgery, they can be associated with right ventricular dysfunction. Heparin-protamine complexes are removed by the reticularendothelial system, and in patients with impaired clearance, an increased protamine concentration (which has a longer half-life compared with heparin) might be observed. Protamine strongly increases vascular resistance in pulmonary arteries, increasing right ventricular afterload, reducing PVR, and leading to cardiac depression and a proarrhythmic state. These effects are linked to protamine-induced liberation of histamine and other pro-inflammatory molecules [25].

\section{Reduction in SVRs (Inappropriate Left Ventricle}

Afterload)

Vasoplegia and/or vasodilatory shock are frequently observed in critically ill patients in the ICU, particularly in those with AKI and multiorgan dysfunction. In these patients, activation of several intrinsic vasodilatory pathways and a reduced vascular responsiveness to vasopressors are frequently observed [26]. Sepsis, in particular, is the most common condition observed in the ICU patients and is one of the most important causes of AKI (often requiring RRT) and hemodynamic instability [27]. The pathophysiologic mechanisms of impaired vascular tone encompass dysregulation of several mediators, such as nitric oxide, inflammatory cytokines, prostaglandins, and complements. In this condition, the impairment of vascular tone occurs at both a precapillary level and a venous level, leading to hemodynamic instability in both systemic arterial vasodilation and venous return (see "A reduction in preload due to absolute or relative hypovolemia") $[7,27]$.

Acidosis and electrolyte disturbances are well-known causes of a reduction in left ventricle afterload. A reduction in perivascular $\mathrm{pH}$ is a well-known cause of vasoplegia. A low $\mathrm{pH}$ reduces calcium influx and inhibits myofilament contractility by inducing smooth muscle cell hyperpolarization. It also reduces the vasoconstrictor response by altering cell surface receptors. In addition to these direct actions of hydrogen ions on smooth muscle, endothelium-derived nitric oxide plays an important role in acidemia-induced hyporesponsiveness to vasoconstrictors. In an acidic environment, nitric oxide is more stable and acidosis may activate nitric oxide synthase [28].

Although changes in potassium concentration due to AKI or RRT might modulate arterial tone [9], calcium is the main electrolyte that clinically restores vascular tone. Dialysate with a higher calcium concentration can increase the extracellular concentration of calcium and can increase arterial tone by direct activation of calcium-sensitive receptors expressed on vascular smooth muscle cells [9].

Beyond these mechanisms, which are typically observed during AKI, other potential mechanisms may induce a pathological reduction in left ventricular afterload during RRT, for example, bioincompatible reactions or extracorporeal clearance of vasopressors. In addition to their cardiac effects, bioincompatible reaction to nonself surfaces can provoke hypotension by decreasing vascular tone (see Fig. 2) and can even lead to vasodilatory shock through the actions of histamine and pro-inflammatory molecules, such as anaphylatoxins, bradykinin, and histamine, on smooth muscle cells $[23,24]$. Finally, a potential cause of hypotension in critically ill patients is clearance vasopressors by RRT. This is not a common mechanism, but it occurs when the venous port of a dialysis catheter lies within the same vein as a separate central venous catheter that is being used for vasopressor infusion and the molecules are cleared prior to exerting their systemic effect [9].

\section{Capillary Barrier and Changes in Solute/Solvent Movement}

Capillary permeability was described by Starling, who included transcapillary oncotic and hydrostatic pressures differences in the same equation. This equation was recently revisited to include another determining factor in the control of the capillary barrier: the glycocalyx. The glycocalyx is a dynamic structure made of a thin layer of proteoglycans, glycoproteins, and other soluble proteins on the endothelial cell surface. The glycocalyx forms the interface between blood and the interstice. Moreover, the glycocalyx plays a role in vasodilation and control of vascular tone. Many pathologic insults, such as sepsis and hypervolemia, can disrupt the glycocalyx and lead to an increase in vascular permeability [6]. AKI and RRT can damage the capillary barrier and alter the microcirculation (see Fig. 1, 2). Glycocalyx impairment leads to alterations in capillary refilling and osmotic shift, which are frequently responsible for hemodynamic instability during aRRT. In particular, glycocalyx dysfunction deadapts fluid transit between the vascular space and the interstitium, limiting physiologic capillary refilling during aRRT. Excessive NetUF that is not compensated by adequate capillary refill promptly results in blood volume reduction and hypotension caused by ventricular preload impairment. However, this may not be the only mechanism 
Table 1. Mechanisms by which aRRT might improve hemodynamic instability

\begin{tabular}{|c|c|c|}
\hline Mechanism & Clinical situation & Improvement \\
\hline \multirow[t]{2}{*}{ Fluid removal } & Volume overload & $\begin{array}{l}\text { Enhanced RV contractility, reduced risk for arrhythmia and organ } \\
\text { dysfunction }\end{array}$ \\
\hline & Acute pulmonary edema & Improvement of RV afterload \\
\hline Filtration of cardiodepressant molecules & Sepsis, trauma & Enhanced cardiac contractility, enhanced vascular tone \\
\hline Removal of DAMPs and PAMPs & Sepsis, trauma & $\begin{array}{l}\text { Improved vascular tone, reduced capillary leak, enhanced response to } \\
\text { vasopressor therapy }\end{array}$ \\
\hline
\end{tabular}

aRRT, acute renal replacement therapy; RV, right ventricle; DAMPs, damage-associated molecular patterns; PAMPs, pathogen-associated molecular patterns.

leading to hypotension after initiation of aRRT. HIRRT can be observed immediately before significant fluid removal, which explains hypotension. Interestingly, even during treatment performed in patients not undergoing NetUF, a reduction in blood volume might be observed during pure dialytic therapy, particularly in those with good clearance who are able to produce an osmotic imbalance between purified blood, tissue interstitium, and intracellular water. Although it is questionable whether the latter mechanism is sufficient to produce HIRRT, it certainly contributes to hemodynamic instability in frail patients with AKI and multiorgan dysfunction treated with aRRT in the ICU. Variation in plasma osmolality depends on pre-RRT osmolality and the degree of solute clearance achieved by aRRT [9].

Critically ill patients are also often hypoalbuminemic. Albumin is the primary contributor to plasma oncotic pressure; thus, hypoalbuminemia may contribute to relative intravascular volume depletion [29]. Patients with hypoalbuminemia have an increased risk of hypotension during maintenance IHD [30]; therefore, albumin infusion has been proposed to prevent or treat HIRRT in these patients. Nonetheless, very little evidence exists in this area and more studies are needed to understand the potential benefits and harms of albumin administration in critical ill patients undergoing RRT [31-33].

\section{ARRT and Restoring Hemodynamic Stability}

Hemodynamic instability has traditionally been attributed to aRRT, particularly when high blood flow and excessive NetUF are prescribed to critically ill patients.
The relationship between a high blood flow and hemodynamic instability has been challenged by various authors [34-36]. This simplistic approach should be re-formulated against the wide consideration of complex and multiple physiologic and pathologic mechanisms that can modulate hemodynamics in patients undergoing aRRT by interacting with $\mathrm{CO}$, peripheral resistance, and the endothelial barrier (see Table 1) [37-39].

\section{Restoration of $\mathrm{CO}$}

\section{Ventricular Preload Optimization}

Considering the epidemiological connection between AKI and hemodynamic instability in critically ill patients, avoiding new or worsened hypotension is particularly challenging when aRRT is applied in the ICU. Considering that these patients are particularly susceptible to preload alterations, a careful assessment of hydration status and fluid responsiveness before aRRT might predict the HIRRT occurrence, but the relationship between predictors of fluid responsiveness and the incidence of HIRRT is still a matter of debate $[9,37,38]$.

Diuretic-refractory volume overload is currently one of the most common indications for aRRT [40]. The application of NetUF in hyperhydrated and frail patients is particularly challenging. In such cases, continuous treatments are the most appropriate in terms of both hemodynamic tolerance and NetUF achieved over time during treatment with IHD and hybrid therapies [41], allowing a lower UF rate but still achieving equivalent fluid removal thanks to a longer treatment time, lower efficiency, and similar intensity. 
Nevertheless, even during CRRT, a careful NetUF should be applied to avoid hemodynamic instability, particularly in patients with inflammation. NetUF should be continuously readjusted during the treatment according to patients' specific pathophysiologic conditions and the status of homeostatic restoration [42].

Excessive NetUF should be avoided to limit a reduction in ventricular preload and hypovolemia. A tailored NetUF protocol might restore hydration status in patients with hypervolemia, particularly in those with an increased preload associated with ventricular dysfunction. In these conditions, sustained NetUF may improve renal perfusion and restore glomerular filtration rate. Interestingly, Murugan et al. [43] found that a high NetUF rate $(>25 \mathrm{~mL} / \mathrm{kg} / \mathrm{day},>1.04 \mathrm{~mL} / \mathrm{kg} / \mathrm{h}$ ) was independently associated with a 1-year reduction in mortality compared with low-intensity NetUF $(<20 \mathrm{~mL} / \mathrm{kg} /$ day $)$ in patients with volume overload, even after adjustment for confounding factors while, in another study, from the same author, a higher NetUf $(>1.75 \mathrm{~mL} / \mathrm{kg} / \mathrm{h})$ was linked to a lower 90-day risk-adjusted survival [44]. A randomized clinical trial is required to validate these findings before they can be applied to clinical practice.

Myocardial Rhythm and Restoration of Contractility Several pathophysiological mechanisms may affect ventricular contractility and rhythm during AKI, such as acidbase alterations, electrolyte disturbances, and inflammation. Interestingly, most of these homeostatic pathways can be restored by adequate RRT. In particular, dialysis has been proven to effectively restore acid-base imbalances in patients with severe metabolic acidosis due to AKI.

Although aRRT itself might cause acid-base alterations (particularly during RCA), a careful, proactive, and methodical approach to aRRT can avoid worsening these alterations [45]. A well-structured pragmatic protocol should be adopted at the bedside during treatment. This protocol should periodically assess buffer mass balance during aRRT delivery and check for citrate accumulation. To check for citrate accumulation, at the very minimum, the total calcium/ionized calcium ratio should be measured daily, and an anticoagulation strategy should be immediately re-discussed if the ratio is $>2.5$. More frequent assessment of this ratio should be considered in specific conditions where hepatic dysfunction or severe shock occur (which may reduce Krebs metabolism), when lactate is increased, during uncontrolled metabolic acidosis, or in patients with systemic ion calcium deficiency requiring calcium compensation, which may be required in cases of impaired metabolism of the calcium-citrate complex.
Beyond acid-base correction, aRRT can be used to restore electrolyte disturbances that lead to cardiac dysfunction, which are mainly potassium and calcium related, in patients with or without AKI. In particular, dialysis can rapidly modulate blood electrolytes with correction directly dependent on the degree of blood disequilibrium. In other words, the higher the blood concentration of these electrolytes, the higher the transmembrane concentration gradient, the higher the transmembrane clearance, and the better the RRT efficiency. For severe acute electrolyte disturbances, highly efficient aRRT (e.g., IHD) may rapidly restore serum calcium and/ or potassium concentrations. The rebound effect frequently observed for blood solutes after a highly efficient treatment should be considered and resolved using maintenance RRT (e.g., CRRT). In such circumstances, the rapid decrease in blood ion concentrations is maintained, despite continuous translocation of the same ionic species from the deep compartments to the blood.

An effective method to achieve ion restoration during RRT is manipulation of dialysate and/or administration of replacement fluids, which should be customized to meet the specific patient's needs. As an example, a dialysate solution with a low concentration of potassium (e.g., $2 \mathrm{mmol} / \mathrm{L}$ ) might rapidly restore hyperkalemia after initiation of aRRT. Nevertheless, if the same solution is applied for maintenance RRT, hypokalemia will be induced. The re-evaluation of dialysate composition and use of solutions with a physiological potassium concentrations (e.g., $4 \mathrm{mmol} / \mathrm{L}$ ), might help physicians to maintain a physiological potassium concentration. The ion concentrations of these solutions should be carefully considered, particularly with RCA. Indeed, a calcium-free dialysate is usually adopted to avoid membrane fouling, while calcium supplementation is directly administered to the patient. Hypocalcemia and hypercalcemia can lead to cardiac dysfunction and hypomagnesemia during RCA.

Beyond the effects on acid-base and electrolyte homeostasis, convective CRRT improves hemodynamics in patients with sepsis and cardiorenal syndrome through several mechanisms, including a reduction in myocardial strain [46] and removal of several cardiodepressant substances. In patients with congestive heart failure, hemofiltration improves CO and cardiac cycle efficiency [47]. Although these effects partly derive from NetUF prescribed in hyperhydrated patients as described above, convective removal of transmembrane cytokines/pathogen-associated molecular patterns/damage-associated molecular patterns seem to counteract myocardial dysfunction in these patients. 
Although slight hypothermia (RRT with dialysate temperature of $35^{\circ} \mathrm{C}$ ) seems to facilitate hemodynamic stability [48], in our opinion euthermia should be achieved, particularly in critically ill patients, because its impacts the microcirculation, oxygen consumption, and membrane excitability, even at the myocardial level, and might positively affect patient outcome. This assumption, however, is mainly based on preclinical evidence and it may be difficult to be tested in an adequately powered randomized trial.

Right ventricular afterload can be reduced by RRT, contributing by restoring $\mathrm{CO}$, particularly in hyperhydrated patients with volume overload refractory to diuretic therapy [49]. In these patients, fluid removal and reduction in extravascular lung water might improve oxygenation [50], reducing hypoxemic pulmonary capillary vasoconstriction and right ventricular afterload. In this context, according to Kidney Disease: Improving Global Outcomes guidelines, heparin-protamine regional anticoagulation should be avoided to reduce the potential detrimental effects that RRT might have in this case on right ventricular afterload [41].

\section{SVR (Left Ventricle Afterload) Restoration}

The effects of RRT on acid-base and electrolyte balance have been described elsewhere. Similar to the positive effects on cardiac function, arterial tone benefited from aRRT via restoration of acid-base and electrolyte balance. In these cases, physiologic restoration of left ventricular afterload might improve oxygen delivery in critically ill patients with AKI, potentially improving global outcomes. Positive effects on arterial tone might be achieved during aRRT through extracorporeal clearance of molecules associated with vasoplegia. Whether based on transmembrane (convective or diffusive) clearance or adsorption, the extracorporeal removal of substances, such as bradykinin, cytokines, nitric oxide, and endotoxins, might restore arterial tone, vasopressor responsiveness, and mean arterial pressure [51]. Finally, the adoption of highly biocompatible disposables might further reduce vasoplegia by avoiding self-activation of the immune system. In particular, polysulfone-based disposables and highly biocompatible heparin-coated surfaces might prevent bioincompatibility reactions, systemic inflammation, and maladaptive vasoplegia.

\section{Capillary Barrier and Solute/Solvent Movement}

We think that continuous aRRT may be an appropriate strategy to provide extracorporeal blood purification in patients with glycocalyx alterations. Considering that systemic inflammation and vasoplegia are usually observed in these patients, continuous therapy might allow physicians to effectively deliver extracorporeal clearancelimit severe hypotension and prevent worsening of multiorgan dysfunction. In particular, a less-intense NetUF protocol applied for a prolonged period of time (theoretically $24 \mathrm{~h}$ ) might allow CRRT to effectively dehydrate patients with volume overload and abnormal capillary refilling, limiting hypotension. Similarly, less-efficient treatments might prevent a clinically evident osmotic shift due to rapid extracorporeal removal of osmotic solutes (e.g., uremic toxins) in patients with an impaired endothelial barrier. Further therapeutic strategies may be adopted to control the decrease in plasmatic osmolality (e.g., dialysate hypertonic solutions and UF or sodium profiling), thus preventing HIRRT [9].

From a physiopathologic point of view, for glycocalyx alterations due to systemic inflammation, extracorporeal blood purification has been proposed to attenuate endothelial dysfunction and mitigate hemodynamic instability. Mainly based on transmembrane clearance and/or on adsorption capacity, purification techniques have been proposed to remove pathogen-associated molecular patterns (e.g., endotoxins) and/or damage-associated molecular patterns (e.g., cytokines) while providing renal support $[51,52]$. Treatments based on transmembrane clearance are usually performed with higher convective doses (e.g., high-volume hemofiltration) or highly porous membranes (e.g., mid- or high-cutoff membranes), although the efficacy of these interventions has not unequivocally been proven to improve patient outcome [53, 54]. Particularly with high-cutoff membranes, the physicians should aim to avoid excessive transmembrane albumin loss, which may occur with more marked convective clearance. This might contribute to glycocalyx alteration, oncotic dysregulation, and hemodynamic instability. Cartridges and hemodiafilters with strong adsorption properties might be limited by biocompatibility issues that further impair endothelial physiology and function.

\section{Conclusions}

A thorough understanding of pathophysiology can guide physicians when prescribing aRRT not only for transient support of renal function, but also to avoid or limit further organ damage and promote faster recovery of organ function. Careful application of aRRT in critically ill patients with AKI could prevent or limit hemodynamic instability. In specific clinical scenarios, hemody- 
namic restoration might be considered an end point of aRRT treatments. A careful multidisciplinary and multiparametric approach to prescribing and delivering aRRT can improve outcomes also in hemodynamically unstable patients with AKI, and a combination of interventions may be more effective than a single intervention. A pathophysiological approach to rectify the main determinants of blood pressure is quintessential to avoid impairments in ventricular preload, contractility, and afterload, as well as rhythm, SVR, and capillary disturbance.

\section{Conflict of Interest Statement}

The authors declare there is no competing interest related to the present manuscript and provide the following disclosures: Gianluca Villa has received support for travel expenses, hotel accommodations, and registration to meetings from Baxter; Stefano Romagnoli has received honoraria for lectures/consultancy from Baxter, Orion Pharma, Vygon, intensive unit medical, MSD, and Medtronic and funds for travel expenses, hotel accommodation, and registration to meetings from Baxter, Pall International, Medigas, and Vygon. All other authors have no disclosures to declare.

\section{Funding Sources}

Authors disclosed that Fondazione Cassa di Risparmio di Firenze (grant number 2014.0679) and "Philip and Irene Toll Gage Foundation" (grant number: na) have economically supported the feasibility and management of this study through research grants aimed at study coordination, statistical analysis, and recruitment of investigators for this study. The funders had no role in study design, data collection and analysis, decision to publish, or preparation of the manuscript.

\section{Author Contributions}

G.V. was responsible for all aspects of the study, thereby ensuring that questions related to the accuracy or integrity of any part of the work were appropriately investigated and resolved. F.H.S. helped with analysis and interpretation of data; he contributed to draft the manuscript and review it critically. T.S. helped with analysis and interpretation of data; he contributed to draft the manuscript and review it critically. D.D.I. helped with analysis and interpretation of data; he contributed to draft the manuscript and review it critically. F.B. helped with analysis and interpretation of data; he contributed to draft the manuscript and review it critically. M.R. helped with analysis and interpretation of data; he contributed to draft the manuscript and review it critically. G.C. helped with analysis and interpretation of data; he contributed to draft the manuscript and review it critically. S.R. substantially contributed to study conception and design, and approved the final version of the manuscript.

\section{References}

1 Malbrain MLNG, Huygh J, Peeters Y, Bernards J. Hemodynamic monitoring in the critically ill: an overview of current cardiac output monitoring methods. F1000Res. 2016; 5:1-9.

2 Girling BJ, Channon SW, Haines RW, Prowle JR. Acute kidney injury and adverse outcomes of critical illness: correlation or causation? Clin Kidney J. 2020;13(2):133-41.

3 Bellomo R, Ronco C, Mehta RL, Asfar P, Boisramé-Helms J, Darmon M, et al. Acute kidney injury in the ICU: from injury to recovery: reports from the 5th Paris International Conference. Ann Intensive Care. 2017;7(1):49.

4 Wang AY, Bellomo R. Renal replacement therapy in the ICU: intermittent hemodialysis, sustained low-efficiency dialysis or continuous renal replacement therapy? Curr Opin Crit Care. 2018;24(6):437-42.

5 Silversides JA, Pinto R, Kuint R, Wald R, Hladunewich MA, Lapinsky SE, et al. Fluid balance, intradialytic hypotension, and outcomes in critically ill patients undergoing renal replacement therapy: a cohort study. Crit Care. 2014;18(6):624-10.

6 Pillinger NL, Kam PCA. Endothelial glycocalyx Endothelial glycocalyx: basic science and clinical implications. Anaesth Intensive Care. 2017;45(3):295-307.
7 Peerapornratana S, Manrique-Caballero CL, Gómez H, Kellum JA. Acute kidney injury from sepsis: current concepts, epidemiology, pathophysiology, prevention and treatment. Kidney Int. 2019;96(5):1083-99.

8 Rangaswami J, Bhalla V, Blair JEA, Chang TI, Costa S, Lentine KL, et al. Cardiorenal syndrome: classification, pathophysiology, diagnosis, and treatment strategies: a scientific statement from the American heart association. Circulation. 2019;139(16):e840-78.

9 Douvris A, Zeid K, Hiremath S, Bagshaw SM, Wald R, Beaubien-Souligny W, et al. Mechanisms for hemodynamic instability related to renal replacement therapy: a narrative review. Intensive Care Med. 2019;45(10):1333-46.

10 Vincent J-L. Fluid management in the critically ill. Kidney Int. 2019;96(1):52-7.

11 Villa G, Samoni S, De Rosa S, Ronco C. The pathophysiological hypothesis of kidney damage during intra-abdominal hypertension. Front Physiol. 2016 Feb;7:1-4.

12 Di Lullo L, Reeves PB, Bellasi A, Ronco C. Cardiorenal syndrome in acute kidney injury. Semin Nephrol. 2019;39(1):31-40.

13 Poole-Wilson PA. Regulation of intracellular $\mathrm{pH}$ in the myocardium; relevance to $\mathrm{pa}$ thology. Mol Cell Biochem. 1989;89(2):1515 .
14 Schneider AG, Journois D, Rimmelé T. Complications of regional citrate anticoagulation: accumulation or overload? Crit Care. 2017; 21(1):281-7.

15 Walker MD, Silverberg SJ. Cardiovascular aspects of primary hyperparathyroidism. J Endocrinol Invest. 2008;31(10):925-31

16 Afshinnia F, Belanger K, Palevsky PM, Young EW. Effect of ionized serum calcium on outcomes in acute kidney injury needing renal replacement therapy: secondary analysis of the acute renal failure trial network study. Ren Fail. 2013;35(10):1310-8.

17 Pun PH, Horton JR, Middleton JP. Dialysate calcium concentration and the risk of sudden cardiac arrest in hemodialysis patients. Clin J Am Soc Nephrol. 2013;8(5):797-803.

18 Di Mario F, Regolisti G, Greco P, Maccari C, Superchi E, Morabito S, et al. Prevention of hypomagnesemia in critically ill patients with acute kidney injury on continuous kidney replacement therapy: the role of early supplementation and close monitoring. J Nephrol. 2020.

19 Ip JE, Cheung JW, Park D, Hellawell JL, Stein KM, Iwai S, et al. Temporal associations between thoracic volume overload and malignant ventricular arrhythmias: a study of intrathoracic impedance. J Cardiovasc Electrophysiol. 2011;22(3):293-9. 
20 Yagi N, Leblanc M, Sakai K, Wright EJ, Paganini EP. Cooling effect of continuous renal replacement therapy in critically ill patients. Am J Kidney Dis. 1998 Dec;32(6):1023-30.

21 Dietrichs ES, Sager G, Tveita T. Altered pharmacological effects of adrenergic agonists during hypothermia. Scand J Trauma Resusc Emerg Med. 2016;24(1):143-9.

22 Slessarev M, Salerno F, Ball IM, McIntyre CW. Continuous renal replacement therapy is associated with acute cardiac stunning in critically ill patients. Hemodial Int. 2019; 23(3):325-32.

23 Davenport A. The role of dialyzer membrane flux in bio-incompatibility. Hemodial Int. 2008;12(Suppl 2):S29-33.

24 Opatrny K. Clinical importance of biocompatibility and its effect on haemodialysis treatment. Nephrol Dial Transplant. 2003 Jul; 18 Suppl 5(90005):v41-4.

25 Sokolowska E, Kalaska B, Miklosz J, Mogielnicki A. The toxicology of heparin reversal with protamine: past, present and future. Expert Opin Drug Metab Toxicol. 2016;12(8) 897-909.

26 Levy B, Fritz C, Tahon E, Jacquot A, Auchet T, Kimmoun A. Vasoplegia treatments: the past, the present, and the future. Crit Care. 2018 Feb;22(1):52.

27 Fani F, Regolisti G, Delsante M, Cantaluppi V, Castellano G, Gesualdo L, et al. Recent advances in the pathogenetic mechanisms of sepsis-associated acute kidney injury. J Nephrol. 2018;31(3):351-9.

28 Celotto AC, Capellini VK, Baldo CF, Dalio $\mathrm{MB}$, Rodrigues AJ, Evora PR. Effects of acidbase imbalance on vascular reactivity. Braz J Med Biol Res. 2008;41(6):439-45.

29 Vincent JL, De Backer D, Wiedermann CJ. Fluid management in sepsis: the potential beneficial effects of albumin. J Crit Care. 2016; 35:161-7.

30 Nakamoto H, Honda N, Mimura T, Suzuki H Hypoalbuminemia is an important risk factor of hypotension during hemodialysis. Hemodial Int. 2006 Oct;10(Suppl 2):S10-5.

31 Hryciw N, Joannidis M, Hiremath S, Callum J, Clark EG. Intravenous albumin for mitigating hypotension and augmenting ultrafiltration during kidney replacement therapy. Clin J Am Soc Nephrol. 2020;CJN.09670620.

32 Gomez H, Priyanka P, Bataineh A, Keener CM, Clermont G, Kellum JA. Effects of 5\% albumin plus saline versus saline alone on outcomes from large-volume resuscitation in critically ill patients. Crit Care Med. 2021; 49(1):79-90.
33 Fortin PM, Bassett K, Musini VM. Human albumin for intradialytic hypotension in haemodialysis patients. Cochrane Database Syst Rev. 2010;(11):CD006758.

34 Ricci Z, Romagnoli S, Ronco C. The 10 false beliefs in adult critical care nephrology. Intensive Care Med. 2018;44(8):1302-5.

35 Eastwood GM, Peck L, Young H, Bailey M, Reade MC, Baldwin I, et al. Haemodynamic impact of a slower pump speed at start of continuous renal replacement therapy in critically ill adults with acute kidney injury: a prospective before-and-after study. Blood Purif. 2012;33(1-3):52-8

36 Trivedi HS, Kukla A, Prowant B, Lim HJ. A study of the extracorporeal rate of blood flow and blood pressure during hemodialysis. $\mathrm{He}$ modial Int. 2007;11(4):424-9.

37 Bitker L, Bayle F, Yonis H, Gobert F, Leray V, Taponnier R, et al. Prevalence and risk factors of hypotension associated with preload-dependence during intermittent hemodialysis in critically ill patients. Crit Care. 2016;20(1): 44-11.

38 Schortgen F, Soubrier N, Delclaux C, Thuong M, Girou E, Brun-Buisson C, et al. Hemodynamic tolerance of intermittent hemodialysis critically ill patients: usefulness of practice guidelines. Am J Respir Crit Care Med. 2000; 162:197-202.

39 Douvris A, Malhi G, Hiremath S, McIntyre L, Silver SA, Bagshaw SM, et al. Interventions to prevent hemodynamic instability during renal replacement therapy in critically ill patients: a systematic review. Crit Care. 2018; 22(1):41-11.

40 Tandukar S, Palevsky PM. Continuous renal replacement therapy: who, when, why, and how. Chest. 2019;155(3):626-38.

41 Kellum JA, Lameire N, Aspelin P, Barsoum RS, Burdmann EA, Goldstein SL, et al. Kidney disease: improving global outcomes (KDIGO) acute kidney injury work group. KDIGO clinical practice guideline for acute kidney injury. Kidney Int Suppl. 2012;2(1):1-138.

42 Doshi M, Murray PT. Approach to intradialytic hypotension in intensive care unit patients with acute renal failure. Artif Organs. 2003;27(9):772-80.

43 Murugan R, Balakumar V, Kerti SJ, Priyanka $\mathrm{P}$, Chang C-CH, Clermont G, et al. Net ultrafiltration intensity and mortality in critically ill patients with fluid overload. Crit Care. 2018;22(1):1-11.
44 Murugan R, Kerti SJ, Chang CCH, Gallagher M, Clermont G, Palevsky PM, et al. Association of net ultrafiltration rate with mortality among critically ill adults with acute kidney injury receiving continuous venovenous hemodiafiltration: a secondary analysis of the randomized evaluation of normal vs. augmented level (RENAL) of renal replacement therapy trial. JAMA Netw Open. 2019;2(6): e195418.

45 Morabito S, Pistolesi V, Tritapepe L, Fiaccadori E. Regional citrate anticoagulation for RRTs in critically ill patients with AKI. Clin J Am Soc Nephrol. 2014;9(12):2173-88.

46 Kotecha A, Vallabhajosyula S, Coville $\mathrm{HH}$, Kashani K. Cardiorenal syndrome in sepsis: a narrative review. J Crit Care. 2018;43:122-7.

47 Giglioli C, Landi D, Cecchi E, Chiostri M, Gensini GF, Valente S, et al. Effects of ULTRAfiltration vs. DIureticS on clinical, biohumoral and haemodynamic variables in patients with deCOmpensated heart failure: the ULTRADISCO study. Eur J Heart Fail. 2011; 13(3):337-46.

48 Edrees FY, Katari S, Baty JD, Vijayan A. A Pilot study evaluating the effect of cooler dialysate temperature on hemodynamic stability during prolonged intermittent renal replacement therapy in acute kidney injury. Crit Care Med. 2019;47(2):e74-80.

49 Lahm T, McCaslin CA, Wozniak TC, Ghumman W, Fadl YY, Obeidat OS, et al. Medical and surgical treatment of acute right ventricular failure. J Am Coll Cardiol. 2010;56(18): 1435-46.

50 Liu H, Liu Y, Sun JK, Xu QL, Yan Y, Chen YM, et al. Extravascular lung water monitoring of renal replacement therapy in lung water scavenging for septic acute kidney injury. Int J Clin Exp Med. 2015;8(10):18907-16.

51 Monard C, Rimmelé T, Ronco C. Extracorporeal blood purification therapies for sepsis. Blood Purif. 2019;47(Suppl 3):2-15 .

52 Honoré PM, Joannes-Boyau O, Gressens B. Blood and plasma treatments: the rationale of high-volume hemofiltration. Contrib Nephrol. 2007;156:387-95.

53 Honore PM, Hoste E, Molnár Z, Jacobs R, Joannes-Boyau O, Malbrain MLNG, et al. Cytokine removal in human septic shock: where are we and where are we going? Ann Intensive Care. 2019;9(1):56.

54 Joannes-Boyau O, Honoré PM, Perez P, Bagshaw SM, Grand H, Canivet JL, et al. Highvolume versus standard-volume haemofiltration for septic shock patients with acute kidney injury (IVOIRE study): a multicentre randomized controlled trial. Intensive Care Med. 2013;39(9):1535-46. 